\title{
Introduction to the Minitrack on Real-World Evaluation of Biosensor-Based Interventions in Healthcare
}

\author{
Edward Boyer \\ Harvard Medical School \\ Edward.boyer@childrens.harvard.edu
}

\author{
Peter Chai \\ Harvard Medical School \\ pchai@bwh.harvard.edu
}

\author{
Stephanie Carreiro \\ University of Massachusetts \\ Medical School \\ Stephanie.carreiro@umassmemorial.org
}

Wearable biosensors have fueled an explosion of novel techniques to detect and respond to disease states through real-time biometric data collection. Detection of activity, worsening disease, and medication adherence through personal fitness trackers, smartphones and advanced medical biosensors provides new insight into the management of diseases and challenges in the security and processing of data.
This minitrack explores real-world deployment and ultization of biosensors among patient populations in addition to concepts and techniques in processing large volumes of biosensor data. Papers accepted for presentation in this minitrack discuss clinically relevant development, deployment and evaluation of technology among patient populations. 